\title{
CONSTRUCTION OF TENSILE STRESS-STRAIN CURVES FOR SOLID BARS PRE-DEFORMED BY GRADIENT SHEAR STRAIN
}

The large strain behavior of metals is usually studied in torsion of cylindrical bars because very large strains can be readily achieved in torsion. Indeed, during tensile testing - which is the most commonly employed characterization technique - the uniform deformation is limited because of early necking. However, the mechanism of strain hardening is quite special for torsion because of the small number of the operating slip systems that lead to smaller equivalent stresses for torsion compared to tension or compression $[1,2]$. This presents difficulties in the characterization of the material behavior at large strains.

The problem of construction of stress-strain curves for torsion of solid bars has been resolved by Fields and Backofen [3] who established a formula for obtaining the flow stress at the outer radius of the twisted bar. However, no such formula is available for tension of a sample with a strain gradient, particularly when a bar is tested in tension after being pre-twisted in torsion. This problem is resolved in the present paper. The importance of such testing is that stress-strain curves can be obtained for tension for very large strains, up to the same strains as in torsion, by tension of pre-twisted bars. For this purpose only thin-walled tubes were pre-twisted so far where the strain gradient can be neglected [4-10]. However, the maximum plastic strain is very limited in torsion of such tubes, which is not the case for the torsion of solid bars.

In the following we first present the theoretical basis and then show that the role of the residual stresses, which are inherent in gradient structures, can be neglected at large strains. Finally, the new technique is applied for the large strain torsion of copper bars.

We consider a solid cylindrical bar twisted in large strain torsion. It can be shown using the equilibrium equation that for large uniform torsion, the local plastic shear strain $\gamma_{r}$ is proportional to the local radius $r$ [11]:

$$
\gamma_{r}=\frac{r}{R} \gamma_{R}
$$

Here $\gamma_{R}$ is the shear strain at the outer radius $(R)$ of the sample. When the twisted bar is subjected to tensile testing, the local tensile flow stress depends on the tensile true strain $\varepsilon$ and on the local shear pre-strain $\gamma_{r} ; \sigma(r)=\sigma\left(\varepsilon, \gamma_{r}\right)$. The force required for plastic stretching of a rod previously subjected by torsion is given by:

$$
F\left(\varepsilon, \gamma_{R}\right)=2 \pi \int_{0}^{R} \sigma\left(\varepsilon, \gamma_{r}\right) r d r
$$

This integral can be developed as follows:

$$
F\left(\varepsilon, \gamma_{R}\right)=2 \pi \int_{0}^{R} \sigma\left(\varepsilon, \frac{r \gamma_{R}}{R}\right) r d r=\frac{2 S}{\gamma_{R}^{2}} \int_{0}^{\gamma_{R}} \sigma\left(\varepsilon, \gamma_{r}\right) \gamma_{r} d \gamma_{r}
$$

where $S$ is the cross-sectional area of the bar. We introduce the quantity $\bar{\sigma}\left(\varepsilon, \gamma_{R}\right)$, which is the apparent tensile flow stress of the bar: 


$$
\bar{\sigma}\left(\varepsilon, \gamma_{R}\right)=\frac{F\left(\varepsilon, \gamma_{R}\right)}{S}=\frac{2}{\gamma_{R}^{2}} \int_{0}^{\gamma_{R}} \sigma\left(\varepsilon, \gamma_{r}\right) \gamma_{r} d \gamma_{r}
$$

After differentiation with respect to $\gamma_{R}$ the following expression is obtained:

$$
\frac{\partial \bar{\sigma}\left(\varepsilon, \gamma_{R}\right)}{\partial \gamma_{R}}=\frac{2}{\gamma_{R}}\left[\sigma\left(\varepsilon, \gamma_{R}\right)-\bar{\sigma}\left(\varepsilon, \gamma_{R}\right)\right] \text {. }
$$

Hence, it follows:

$$
\sigma\left(\varepsilon, \gamma_{R}\right)=\bar{\sigma}\left(\varepsilon, \gamma_{R}\right)+\frac{\gamma_{R}}{2} \frac{\partial \bar{\sigma}\left(\varepsilon, \gamma_{R}\right)}{\partial \gamma_{R}}
$$

This formula allows one to find the stress-strain curve $\sigma=\sigma\left(\varepsilon, \gamma_{R}\right)$ using the experimentally measured curve for the apparent stress $\bar{\sigma}\left(\varepsilon, \gamma_{R}\right)$ and its derivative with respect to $\gamma_{R}$.

Residual stresses arise after unloading a plastically twisted solid bar sample [11]. Assuming that the entire cross section of the sample was in plastic state under torsion, the local residual shear stress $\tau_{\text {res }}\left(r, \gamma_{R}\right)$ after a shear strain $\gamma_{R}$ reached at the outer radius of the bar is given by the following equation [12]:

$$
\tau_{\text {res }}\left(r, \gamma_{R}\right)=\tau\left(r, \gamma_{R}\right)-\frac{4}{3} \frac{r}{R} \bar{\tau}\left(\gamma_{R}\right)
$$

Here $\tau\left(r, \gamma_{R}\right)$ is the local shear flow stress before unloading and $\bar{\tau}$ is the mean shear stress across the bar:

$$
\bar{\tau}\left(\gamma_{R}\right)=\frac{3}{R^{3}} \int_{0}^{R} \tau\left(r, \gamma_{R}\right) r^{2} d r
$$

The effect of the residual stresses during the subsequent tensile testing is a reduction of the yield stress of the rod in tension, as they are present in the yield condition. It can be shown, however, that the residual stresses can be ignored when the technique presented above is applied. This is due to a rapid relaxation of the residual stresses at the beginning of plastic deformation. As will be shown below, the strain required for this relaxation is very small. The residual elastic strain $\gamma_{\text {res }}$ associated to the residual elastic stress $\tau_{\text {res }}$ is:

$$
\gamma_{\text {res }}=\frac{\tau_{\text {res }}}{G}
$$

where $G$ is the elastic shear modulus. According to the associated flow rule, the following relation between the components of the strain increments and the acting stresses is valid:

$$
d \gamma=\frac{3 \tau_{r e s}}{\sigma} d e_{V M}
$$

where $e_{V M}$ is the von Mises equivalent strain. It follows then from Eqs. (9) and (10) that

$$
d \gamma_{\text {res }}=-\gamma_{\text {res }} \frac{3 G}{\sigma} d e_{V M}
$$


Here the negative sign takes into account that $\gamma_{\text {res }}$ decreases in absolute value, so that the sign of $d \gamma_{\text {res }}$ is opposite to the sign of $\gamma_{\text {res }}$. By integrating Eq. (11) at a constant stress $\sigma$, we obtain:

$$
\gamma_{\text {res }} \sim \exp \left(-\frac{3 G}{\sigma} e_{V M}\right)
$$

Therefore, the characteristic equivalent plastic strain $e_{V M}$ required for the relaxation of residual stresses for torsion-tension can be estimated as

$$
e_{V M}=\frac{\sigma}{3 G} \text {. }
$$

Using characteristic stress values that correspond to the tensile test of copper after torsion, the following estimate is obtained: $e_{V M} \sim 10^{-3}$. This estimate shows that already after a very small tensile strain, the effect of the residual stresses can be neglected. The physical meaning of the above analysis is that the stress state moves along the yield surface very rapidly from a combined tensiontorsion into a pure tension state during the initial stage of the tensile test.

Experiments were carried out on commercially pure copper samples at room temperature. The initial microstructure of the material can be characterized by an average grain size of about $30 \mu \mathrm{m}$ with a weak crystallographic texture. The dimensions of the deforming part of the samples were as follows: $7 \mathrm{~mm}$ in diameter and $40 \mathrm{~mm}$ in gauge length. The torsion testing was done in a free-end torsion machine to different rotation angles at a constant angular speed of $0.2 \mathrm{rad} / \mathrm{s}$. The selected values of the rotation angle (in radian) were: 11.43, 22.85, 34.29, 45.71, 57.14, and 68.57. They correspond, respectively, to a shear strain of $1,2,3,4,5$, and 6 , at the outer radius of the sample. These values were converted into the von $M$ ises equivalent strain using the formula $e_{V M}=\gamma / \sqrt{3}$. The small lengthening of the bar during the free end torsion testing (less than $2 \%$ ) was neglected in the analysis of the experimental data. The shear flow stress acting at the outer radius of the bar was calculated by the Fields and Backofen formula [3] and converted into equivalent von Mises stress using the formula $\sigma=\sqrt{3} \tau$. The obtained stress-strain curve for torsion curve is displayed in Fig. 1 (curve No. 2).

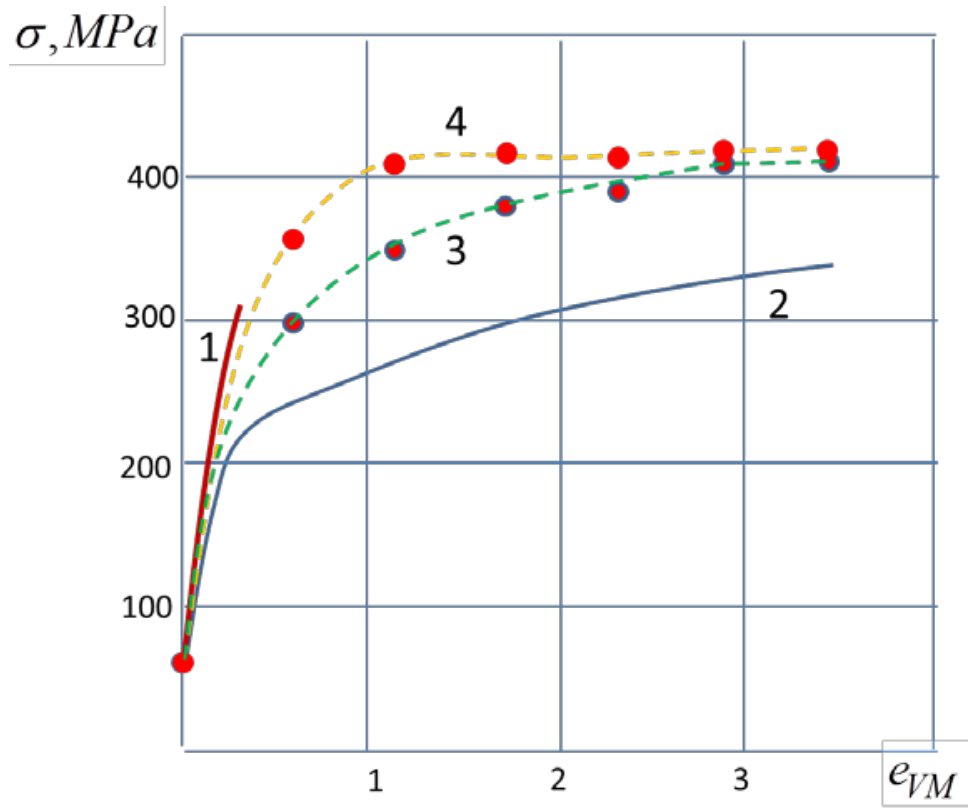

Fig. 1. Stress-strain curves obtained for pure copper solid bars in tension (curve No. 1), in torsion (curve no. 2) and in tension after torsion with different magnitude of the twist (curve No. 3 ). Curve No. 4 was constructed using Eq. (6) 
The tensile tests were done in a 10 ton Zwick machine at a strain rate of $0.05 \mathrm{~mm} / \mathrm{s}$. The results are shown in Fig. 2 for the twisted samples and in Fig. 1 for the non twisted ones for larger strain (curve No. 1). After torsion rupture took place under tension already after about $2-4 \%$ plastic strain. With the available specimens, six points on the average stress-strain curve were obtained. This is labelled No. 3 in Fig. 1. They were taken from the tensile curves at $1 \%$ strain. A continuous curve was fitted to these points to calculate the derivative in Eq. (6), and the resulting large strain tensile test curve base on Eq. (6) was plotted as curve No. 4. It can be seen that the initial part of this curve matches the continuous tensile curve well. At large deformations, from about a strain of 1.5, the curve levels off at a constant stress level of about $415 \mathrm{MPa}$.

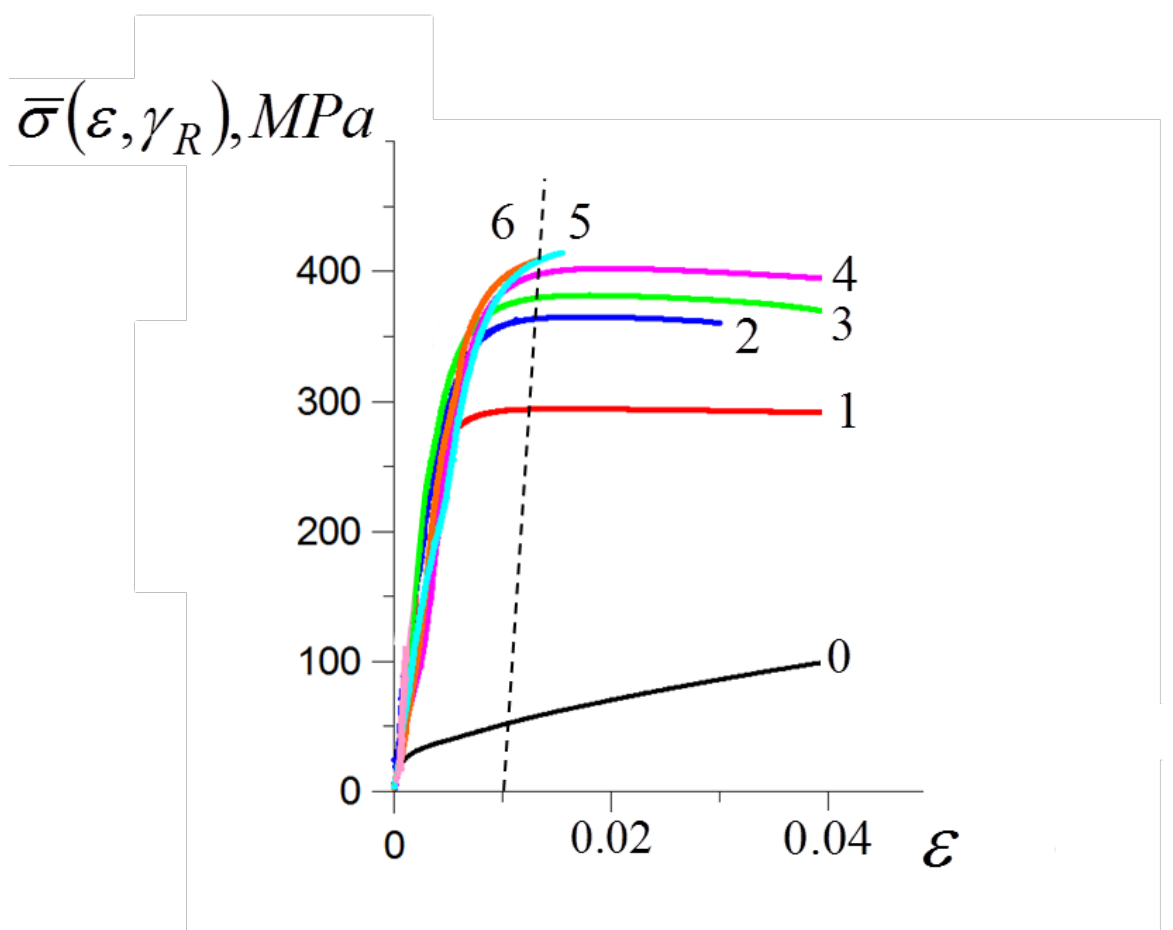

Fig. 2. Stress-strain curves in tension obtained without pre-torsion (0) and after different amounts of shear in torsion $(1: \gamma=1,2: \gamma=2,3: \gamma=3,4: \gamma=4,5: \gamma=5,6: \gamma=6)$.

The stress-strain curve No. 4 in Fig. 1 obtained for tensile testing after torsion was constructed for the material behavior at the outer radius of the twisted bar, but it is also valid for the inner points in the bulk of the bar.

As can be seen from Fig. 1, the monotonic torsion and tensile stress-strain curves do not coincide, despite the use of the equivalent stress and strain quantities as a common platform: the torsion curve lies below the tensile one. This effect known for a long time and was examined in the past [1, 2]. The main reason for it was mentioned in Section 1, viz. the scarcity of slip systems in torsion compared to tension.

One particularity of the present results is that for low tensile strains the tensile flow stress after torsion agrees well with the flow stress in monotonic tension. Indeed, lower stresses are expected for a strain path change because the microstructure that develops in the first path is not stable for the new path, thus many dislocations that were immobile in the first path can glide in the second one. However, it has been shown in Ref. [2] that the dislocation density is higher in torsion compared to tension, which can compensate for this effect.

Another particularity of the results in Fig. 1 is that the tensile flow stress is constant after torsion at large strains, starting from about 1.5 strain. This effect was observed for the first time because the present technique is the first one to provide access to tensile flow stress after large strain torsion. Its origin might be rooted in the fragmentation of the grains which is occurs under severe plastic deformation [13]. Further studies are needed to identify the exact reasons for this material behavior. 


\section{CONCLUSIONS}

One particularity of the present results is that for low tensile strains the tensile flow stress after torsion agrees well with the flow stress in monotonic tension.

Another particularity of the results is that the tensile flow stress is constant after torsion at large strains, starting from about 1.5 strain. This effect was observed for the first time because the present technique is the first one to provide access to tensile flow stress after large strain torsion.

\section{REFERENCES}

1. Tome C., Canova G.R., Kocks U.F., Christodoulou,N., Jonas J.J., The Relation Between Macroscopic and Microscopic Strain Hardening in F.C.C. Polycrystals. Acta Metall. 1984. 32, pp. 1637-1653.

2. Ungar T., Toth L.S., Illy J., Kovacs I. Dislocation-structure and work hardening in polycrystalline OFHC copper rods deformed by torsion and extension. Acta Metall. 1986. 34, pp. 1257-1267.

3. Fields Jr D.S., Backofen W.A. Determination of Strain Hardening Characteristics by Torsion Testing. Proc. Am. Soc. Test. Mater. 1957. 57, pp. 1259-1272.

4. Ilyushin A.A., Lensky V.S. 1967. Strength of Materials, Pergamon Press, Oxford.

5. Bell J., Khan A.S. Finite Plastic Strain in Annealed Copper During Non-Proportional Loading. Int. J. Solids Structures. 1980. 16, pp. 683-693.

6. Delobelle P., Robinet P., Geyer P., Bouffioux P. A model to describe the anisotropic viscoplastic behaviour of Zircaloy-4 tubes. J. Nuclear Mater. 1996. 238, pp. 135-162.

7. Takahashi H., Fujiwara K., Nakagawa T. Multiple-slip work-hardening model in crystals with application to torsion-tension behaviors of aluminium tubes. Int. J. Plasticity. 1998. 14, pp. 489-509.

8. Khan A.S., Liang R. Behaviors of three BCC metals during non-proportional multi-axial loadings: experiments and modeling. Int. J. Plasticity. 2000. 16, pp. 1443-1458.

9. Faleskog J., Barsoum I. Tension-torsion fracture experiments-Part I: Experiments and a procedure to evaluate the equivalent plastic strain. Int. J. Solids and Structures. 2013. 50, pp. 4241-4257.

10. Mehrabi R., Andani M.T., Kadkhodaei M., Elahinia M. Experimental Study of NiTi Thin-Walled Tubes Under Uniaxial Tension, Torsion, Proportional and Non-Proportional Loadings. Experimental Mechanics. 2015. 55, pp. 1151-1164.

11. Mehrabi R., Andani M.T., Kadkhodaei M., Elahinia M. Experimental Study of NiTi Thin-Walled Tubes Under Uniaxial Tension, Torsion, Proportional and Non-Proportional Loadings. Experimental Mechanics. 2015. 55, pp. 1151-1164.

12. Jonas J.J. Montheillet F. Shrivastava S. The elastic unloading of torsion bars subjected to prior plastic deformation. Scripta Metall. 1985. 19, pp. 235-240.

13. Valiev R.Z., Estrin Y., Horita Z., Langdon T.G., Zehetbauer M.J., Zhu Y.T. Producing bulk ultrafinegrained materials by severe plastic deformation. JOM. 2006. 58, pp. 33-39.

Cai Chen - Ph.D., Researcher DAMAS

Yan Beygelzimer - Professor, Principal Researcher DIPhE NASU

Roman Kulagin - Ph. D., Researcher INT, KIT

Oleksandr Davydenko - Ph.D., Senior Researcher DIPhE NASU

DAMAS - Laboratory of Excellence on Design of Alloy Metals for low-mAss Structures, Université de Lorraine, Metz, France.

DIPhE NASU - Donetsk Institute for Physics and Engineering named after O. O. Galkin, National Academy of Sciences of Ukraine, Kyiv.

INT, KIT - Institute of Nanotechnology, Karlsruhe Institute of Technology, Germany.

E-mail: yanbeygel@gmail.com

Received 06.02.2020. 